\title{
Niveles de resiliencia y la presencia de síntomas depresivos en los estudiantes universitarios
}

\author{
Resilience levels and the presence of depressive symptoms in university students
}

Níveis de resiliência e presença de sintomas depressivos em estudantes universitários

Jisson Oswaldo Vega Intriago

jissonosvein@gmail.com

https://orcid.org/0000-0001-5727-8837

Universidad Técnica de Manabí, Portoviejo, Ecuador
Carmen Liliana Borrero Pinargote

cborrero2040@utm.edu.ec

https://orcid.org/0000-0002-8707-885X

Universidad Técnica de Manabí, Portoviejo, Ecuador

Edith Madeleine Cedeño Mendoza

eceden00206@utm.edu.ec

https://orcid.org/0000-0001-8003-3635

Universidad Técnica de Manabí, Portoviejo, Ecuador

Recibido: febrero 2021 / Revisado: marzo 2021 / Aceptado: abril 2021 / Publicado: mayo 2021

RESUMEN

La investigación tuvo como objetivo identificar los niveles de resiliencia y síntomas depresivos en los estudiantes de la carrera de psicología de la Universidad Técnica de Manabí, afectados por el estado de emergencia sanitaria y humanitaria asumida en Ecuador, ante la presencia del COVID-19 durante el periodo de Junio- Julio 2020. Desde un paradigma mixto (cuali cuantitativo) se empleó el método inductivo-deductivo aplicando el empleo de la escala de resiliencia de Saavedra y Villalta, del inventario de depresión de Beck II y de la entrevista semiestructurada; asistidos por el Coeficiente Correlacional de Pearson para identificar la relación directa que existe entre las variables mencionadas. Los resultados evidenciaron que a mayor resiliencia menores son los síntomas depresivos. Se concluye que considerando la situación universal del comportamiento de la pandemia, se corre el riesgo de revertir la situación, generando la disminución de la resiliencia y por consecuencia el aumento de síntomas depresivos.

Palabras clave: Resiliencia; depresión; correlación; pandemia
ABSTRACT

The objective of the research was to identify the levels of resilience and depressive symptoms in psychology students at the Technical University of Manabí, affected by the state of health and humanitarian emergency assumed in Ecuador, in the presence of COVID-19 during the June-July 2020 period. From a mixed paradigm (qualitative quantitative) the inductive-deductive method was used applying the use of the Saavedra and Villalta resilience scale, the Beck II depression inventory and the semi-structured interview; assisted by Pearson's Correlational Coefficient to identify the direct relationship that exists between the mentioned variables. The results showed that the greater the resilience, the lower the depressive symptoms. It is concluded that considering the universal situation of the behavior of the pandemic, there is a risk of reversing the situation, generating a decrease in resilience and consequently an increase in depressive symptoms.

Key words:

Resilience; depression; correlation; pandemic
RESUMO

O objetivo da pesquisa foi identificar os níveis de resiliência e sintomas depressivos em estudantes de psicologia da Universidade Técnica de Manabí, afetados pelo estado de saúde e emergência humanitária assumida no Equador, na presença do COVID-19 durante o período junho-julho Período 2020. A partir de um paradigma misto (qualitativo quantitativo) utilizou-se o método indutivo-dedutivo aplicandose o uso da escala de resiliência de Saavedra e Villalta, o inventário de depressão de Beck II e a entrevista semiestruturada; auxiliado pelo Coeficiente Correlacional de Pearson para identificar a relação direta que existe entre as variáveis mencionadas. Os resultados mostraram que quanto maior a resiliência, menores são os sintomas depressivos. Conclui-se que, considerando a situação universal do comportamento da pandemia, existe o risco de reversão da situação, gerando diminuição da resiliência e, consequentemente, aumento dos sintomas depressivos.

Palavras-chave: Resiliência; depressão; correlação; pandemia 


\section{INTRODUCCIÓN}

A partir de la situación de emergencia sanitaria y humanitaria que se vive actualmente a causa de la pandemia de COVID-19, se han llevado a cabo muchas investigaciones que muestran la presencia de síntomas depresivos; entre otro tipo de sintomatología psicológica con las diferencias relativas a cada lugar investigado.

El grupo de psicólogos de la Universidad Complutense de Madrid en abril de 2020, tuvo como resultado que uno de cada cinco españoles presentaron significativamente síntomas depresivos (Viejo, 2020) En una investigación realizada en la comunidad autónoma Vasca del Norte de España en 2020, se concluyó que si bien la sintomatología es baja de manera general, la población más joven presenta mayores niveles que el resto (Ozamiz, Dosil, Picaza e Idoiaga, 2020).

En Estados Unidos, el Gobierno realizó en el presente año una investigación concluyendo que los adultos jóvenes, son quienes presentan mayor gravedad, también las mujeres y la población de escasos recursos (Infobae, 2020).

Schofield, O'Halloran, Mclean y ForresterKnauss (2016) en la Universidad Metropolitana de Victoria en Australia, identificaron que la resiliencia se asocia negativamente a la conducta depresiva en los estudiantes. Por lo que recomienda que las autoridades encargadas de la educación superior promocionen la salud mental y propicien el desarrollo de la resiliencia en la población universitaria.

En base a estos resultados se puede notar la importancia de promover la resiliencia, puesto que actúa como amortiguador psicoemocional ante los momentos de adversidad. Especialmente en la población con sintomatología más acentuada que comprende la etapa de la juventud.

En Ecuador, durante los meses de marzo y abril de 2020 se llevó a cabo una investigación por la Universidad San Francisco de Quito
(USFQ) y la Escuela Politécnica Nacional (EPN) en la cual se entrevistaron a 3879 personas de las diferentes ciudades del país, para conocer sobre temas diversos alrededor de la pandemia como causa de la salud mental. Uno de los resultados del estudio es que el estado de ánimo de los ecuatorianos se ha visto impactado como consecuencia del aislamiento y la cuarentena, siendo las mujeres las más afectadas. Tanto a hombres como a mujeres se les ha incrementado los niveles de ansiedad en los últimos meses. Otro aspecto relevante es que quienes han presentado mayormente esta problemática es la población que comprende entre los 18 y 36 años (El Universo, 2020) Estos resultados obtenidos coinciden con las investigaciones anteriormente expuestas, en tanto que es la población adulta joven, la que presenta mayores síntomas depresivos a consecuencia de la situación vivida por la pandemia.

En el ser humano predomina la afectividad, por eso es ineludible que dentro de su estado emocional y ante ciertas situaciones adversas, se presenten la desesperanza, la tristeza, la anhedonia, el miedo, entre otros síntomas que afectan su funcionalidad. Por fortuna estos síntomas depresivos se enfrentan con la resilencia como amortiguador que de alguna manera los mitiga.

La Organización Mundial de la Salud (OMS, 2020) consideró que la depresión es un trastorno mental frecuente, que se caracteriza por la presencia de síntomas como lo es la tristeza, pérdida de interés o placer, sentimientos de culpa o falta de autoestima, trastornos del sueño o del apetito, sensación de cansancio y falta de concentración.

Por el contrario la resiliencia es una capacidad que permite afrontar situaciones adversas, de tal manera que proporcione un cambio positivo en la vida de las personas. La resiliencia es el resultado de la interacción de ciertos factores protectores intrínsecos y externos al ser humano. Saavedra (2008) menciona que una conducta resiliente 
se ve influenciada por algunos elementos como los temporales, espaciales, histórico y sociales. Entonces la resiliencia es fruto de la interacción entre factores de riesgo y factores protectores, los cuales se encuentran presentes tanto en el medio como en el sujeto mismo.

Como un factor externo se puede mencionar el brote pandémico que ha provocado incertidumbre entre los habitantes del mundo, por la propagación rápida y el peligro que implica el contraer este virus que ya se ha cobrado muchas vidas.

El nuevo coronavirus se llama SARS-CoV2, la enfermedad se llama Corona Virus Disease 2019=COVID19, es un nuevo tipo de coronavirus que puede afectar a las personas y se ha detectado por primera vez en diciembre de 2019 en la ciudad de Wuhan, provincia de Hubei, en China. Todavía hay muchas cuestiones que se desconocen en relación a la enfermedad que produce: COVID-19.

Ante la presente crisis sanitaria hay cabida para la preocupación, misma que se presenta al igual que en todas las personas, en los estudiantes universitarios, llegando al punto de manifestar síntomas depresivos que pueden menoscabar su integridad.

López (2015) refiere que diversos sentimientos como incertidumbre, inseguridad, ansiedad, tristeza o enfado, se presentan en esta población estudiantil. Se produce una crisis como consecuencia del cambio en la rutina diaria y el aislamiento que obliga a una rápida adaptación. Cada persona podrá enfrentar esta situación de forma diferente según su capacidad de resiliencia para soportar presiones, adversidades y traumas. Pero no se trata solo de fomentar la resiliencia individual, existen entornos facilitadores que hay que fortalecer. La contención social de la familia, los amigos y los equipos de salud quienes favorecen que esta situación se transite lo mejor posible.

Esta investigación tuvo como finalidad conocer los niveles de Resiliencia de los estudiantes de psicología. También se evaluó la presencia de síntomas depresivos y la relación entre las variables mencionadas, dando respuesta a la siguiente interrogante ¿Actúa la resiliencia como factor protector frente a la presencia de síntomas depresivos en los estudiantes de psicología de la Universidad Técnica de Manabí, afectados por el estado de emergencia sanitaria y humanitaria en Ecuador por la presencia del COVID-19 durante el periodo de Junio -Julio 2020? Por tal motivo se redactaron los objetivos de investigación siguientes:

\section{General:}

- Identificar los niveles de resiliencia y la presencia de síntomas depresivos en los estudiantes de la carrera de psicología de la Universidad Técnica de Manabí, afectados por el estado de emergencia sanitaria y humanitaria en Ecuador por la presencia del COVID-19 durante el periodo de Junio- Julio 2020.

\section{Específicos:}

- Medir los niveles de resiliencia de los estudiantes de psicología de la Universidad Técnica de Manabí, afectados por el estado de emergencia sanitaria y humanitaria en Ecuador por la presencia del COVID-19 durante el periodo de Junio-Julio 2020.

- Evaluar la presencia de síntomas depresivos en los estudiantes de la carrera de psicología de la Universidad Técnica de Manabí, afectados por el estado de emergencia sanitaria y humanitaria en Ecuador por la presencia del COVID-19 durante el periodo de Junio - Julio 2020.

- Analizar la relación entre las variables resiliencia y síntomas depresivos en la población mencionada anteriormente. 
Es preciso destacar en la marco de la justificación del presente estudio que las consecuencias psicológicas generadas por la crisis sanitaria y humanitaria en Ecuador son cada vez más evidentes, siendo notoria la población universitaria, debido que al enfrentarse a esta situación desconocida se le presenta una incertidumbre por el futuro profesional, trayendo consigo secuelas en el plano psicológico y emocional. Razón por la que es importante desde el plano psicoemocional investigar el tema propuesto, ya que permitió establecer la incidencia de la resiliencia como factor protector ante la presencia de síntomas depresivos generados por la presencia de la pandemia actual.

Esta investigación precisó si los estudiantes universitarios de psicología poseen sintomatología depresiva, a la vez identificó si la resiliencia actúa de forma determinante para prevenir los síntomas depresivos.

La realización de esta investigación fue viable, ya que se dispuso de los recursos tanto humanos, materiales, tecnológicos y económicos. Se llevó a cabo con la participación de 100 estudiantes bajo la modalidad virtual, a través de la herramienta Formularios de Google Drive. Los datos recabados propiciaron el conocimiento de información pertinente tanto para el ámbito estudiantil como psicosocial. La COVID 19 trajo consigo algunas consecuencias enormes para la sociedad en general, debido a que obliga a un gran cambio de la normalidad que conocíamos, y con ello la adaptación a la nueva realidad estudiantil.

\section{MÉTODO}

A partir de la relación pandemia, depresión, resiliencia; y con el auxilio de las técnicas prueba psicométricas y entrevista semiestructurada, se aplicó el método inductivo-deductivo para observar cómo ciertos fenómenos están asociados, y se intentó descubrir la ley o los principios que permiten dicha asociación. Fue pertinente el empleo de los Métodos de Coeficiente Correlacional de Pearson para identificar la relación directa que existe entre las variables ya mencionadas.

Otros instrumentos que asistieron fueron la Escala de Resiliencia SV-RES, la cual permitió establecer un nivel de resiliencia de manera general, además de conocer los factores específicos que lo conforman para trabajar sobre ellos. El Inventario de Depresión de Beck-II, es un cuestionario auto administrado que consta de 21 preguntas de respuestas múltiples para medir la presencia de síntomas depresivos y que tan severa puede ser una depresión.

La población objeto de estudio fueron estudiantes de la carrera de psicología de la Universidad Técnica de Manabí y para la muestra se consideraron al azar 100.

Los resultadosobtenidos mediantelaaplicación de los distintos instrumentos, permitieron demostrar que a mayor resiliencia, los síntomas depresivos que se pueden generar por el estado de emergencia sanitaria y humanitaria en Ecuador ante la presencia del COVID-19, disminuyen; por el contrario si esta capacidad se acorta aumentan los síntomas depresivos.

\section{RESULTADOS}

Los resultados fueron obtenidos gracias a la herramienta de Google Drive formularios, a través de la cual fueron digitalizados los 3 instrumentos utilizados para la investigación. Cada link fue enviado a la muestra, luego se realizó la calificación de la escala de resiliencia de Saavedra y Villalta y del inventario de depresión de Beck II. La entrevista fue sometida a un análisis cualitativo y cuantitativo. Finalmente se aplicó la fórmula para encontrar el coeficiente correlacional de Pearson, dando de esta manera respuesta a los objetivos planteados inicialmente por las autoras. (Tabla 1 ). 
Tabla 1. Escala de resiliencia de Saavedra y Villalta.

\begin{tabular}{llcc}
\hline \multicolumn{1}{c}{ Puntaje directo } & \multicolumn{1}{c}{ Puntajes en valor percentil } & Frecuencia & Porcentaje \\
\hline $0-235$ & Puntaje percentil 0 - 25 = Bajo. & 14 & $14 \%$ \\
$236-274$ & Puntaje percentil 26 - 74 = Promedio. & 44 & $44 \%$ \\
$275-300$ & Puntaje percentil 75 - 99 = Alto. & 42 & $42 \%$ \\
& & $\mathbf{1 0 0}$ & $\mathbf{1 0 0 \%}$ \\
\hline
\end{tabular}

\section{Análisis e interpretación}

En base a la recopilación y posterior tabulación de datos se obtuvo que 14 personas estuvieron en un nivel bajo de la escala de resiliencia; 44 personas, obtuvieron puntuaciones que se consideran promedio y finalmente 42 personas consiguieron una calificación que los ubica en un nivel alto.

A partir de esto se analizó que casi la mitad de la población, es decir 44 estudiantes mantienen niveles promedios de resiliencia, y otra gran parte de la población equivalente a 42 un puntaje alto. Si se suman estos dos valores la mayoría de la población posee características que potencian sus niveles, a diferencia de un grupo de 14 personas que se encuentran con un nivel bajo. (Tabla 1 ).

Tabla 2. Inventario de Depresión de Beck II.

\begin{tabular}{lcc}
\hline \multicolumn{1}{c}{ Puntuaciones } & Frecuencia & Porcentaje \\
\hline 0-13, mínima depresión & 79 & $79 \%$ \\
14-19, depresión leve & 13 & $13 \%$ \\
20-28, depresión moderada & 6 & $6 \%$ \\
29-63, depresión grave & 2 & $2 \%$ \\
Total & $\mathbf{1 0 0}$ & $\mathbf{1 0 0 \%}$ \\
\hline
\end{tabular}

\section{Análisis e interpretación}

Una vez recopilados y tabulados los datos se diría que a partir de la aplicación del inventario de depresión de Beck II, se conoció la sintomatología depresiva en la muestra. 79 estudiantes se encuentran en el nivel de síntomas mínimos de depresión; 13 personas obtuvieron puntajes que los ubica en el rango de sintomatología de depresión leve; 6 estudiantes se encuentran en el intervalo que representa síntomas de depresión moderada y finalmente 2 estudiantes obtuvieron puntajes que los encaja en el nivel de sintomatología de depresión grave. (Tabla 2).

\section{Entrevista semiestructurada}

Para efectos operativos solo se tomaron algunos ítems de la totalidad de la entrevista.

¿Ha sentido en las últimas dos semanas sentimientos de tristeza o desconsolación? 
Tabla 3. Sentimientos de tristeza.

\begin{tabular}{lcc}
\hline \multicolumn{1}{c}{ Opciones } & Frecuencia & Porcentajes \\
\hline Si, muy a menudo & 9 & $9 \%$ \\
Si, algunas veces & 33 & $33 \%$ \\
Sí, pero muy poco & 28 & $28 \%$ \\
No & 30 & $30 \%$ \\
TOTAL & 100 & $\mathbf{1 0 0 \%}$ \\
\hline
\end{tabular}

\section{Análisis e interpretación}

A la interrogante sobre si han tenido sentimientos de tristeza 9 personas expresaron que sí, muy a menudo; 33 personas mencionaron que sí, pero algunas veces; 28 personas respondieron que sí, pero muy poco y finalmente 30 personas, respondieron que no. Si se suman las tres primeras opciones resulta que 70 personas es decir la mayoría si ha presentado sentimientos de tristeza o desconsolación. (Tabla 3).

Se suma la pregunta ¿En las últimas dos semanas ha experimentado fatiga o pérdida de energía?

Tabla 4. Sensación de fatiga.

\begin{tabular}{lccc}
\hline & Opciones & Frecuencia & Porcentajes \\
\hline Siempre & 8 & $8 \%$ \\
Casi siempre & 15 & $15 \%$ \\
Algunas veces & 27 & $27 \%$ \\
Pocas veces & 30 & $30 \%$ \\
Nunca & 20 & $20 \%$ \\
Total & 100 & $\mathbf{1 0 0 \%}$ \\
\hline
\end{tabular}

\section{Análisis e interpretación}

En cuanto a esta interrogante, 8 personas manifestaron que siempre sintieron fatiga 0 pérdida de energía; 15 casi siempre; 27 algunas veces; 30 personas respondieron que pocas veces y 20 personas respondieron que nunca sintieron perdida de energía durante las últimas dos semanas. (Tabla 4).

$\mathrm{Si}$ se suman las 4 primeras opciones que representan el $80 \%$, se evidencia que sí se presentó en alguna medida pérdida de energía o fatiga, solo con algunas variaciones en cuanto a su temporalidad.

Otra interrogante del proceso investigativo fue: ¿En las últimas dos semanas ha experimentado alteracoines en la conducta del sueño, como insomnio, problemas en el sueño o duerme de manera excesiva? 
Tabla 5. Problemas de sueño.

\begin{tabular}{lccc}
\hline & Opciones & Frecuencia & Porcentajes \\
\hline Siempre & 14 & $14 \%$ \\
Casi siempre & 15 & $15 \%$ \\
Algunas veces & 21 & $21 \%$ \\
Pocas veces & 27 & $27 \%$ \\
Nunca & 23 & $23 \%$ \\
Total & $\mathbf{1 0 0}$ & $\mathbf{1 0 0 \%}$ \\
\hline
\end{tabular}

\section{Análisis e interpretación}

Catorce personas manifestaron que siempre presentan síntomas de alteración en el sueño; 15 casi siempre; 21 algunas veces; 27 pocas veces y finalmente 23 respondieron que nunca presentaron este tipo de conducta. (Tabla 5).

Se observó una frecuencia menor en la opción de siempre, la opción que mayor frecuencia obtuvo fue la de pocas veces. Esto dejó ver que en pocas ocasiones estas conductas de alteración del sueño estuvieron presentes en la población de estudio.

Otra pregunta de la ruta de estudio fue ¿En las últimas dos semanas ha experimentado falta de placer en actividades que antes encontraba satisfactorias?

Cuadro 6. Experiencias placenteras.

\begin{tabular}{lccc}
\hline & Opciones & Frecuencia & Porcentajes \\
\hline Sí & 40 & $40 \%$ \\
No & 60 & $60 \%$ \\
Total & 100 & $\mathbf{1 0 0 \%}$ \\
\hline
\end{tabular}

\section{Análisis e interpretación}

Cuarenta personas mencionaron si haber presentado anhedonia mientras que el 60 expresaron que no. Sin embargo el 40 es un valor significativo, pues casi la mitad de la población si presentó falta de placer en actividades que antes encontraba satisfactorias. (Tabla 6).
Como cierre se tomó en cuenta ¿En que medida atribuye la presencia de su estado emocional actual al aislamiento social y las nuevas formas de trabajo y estudio a las que debemos adaptarnos los ecuatorianos?

Cuadro 7. Problemas de sueño.

\begin{tabular}{lccc}
\hline & Opciones & Frecuencia & Porcentajes \\
\hline Mucho & 52 & $52 \%$ \\
Poco & 40 & $40 \%$ \\
Nada & 8 & $8 \%$ \\
Total & 100 & $\mathbf{1 0 0 \%}$ \\
\hline
\end{tabular}




\section{Análisis e interpretación}

Cincuenta y dos personas respondieron que atribuyen mucho; 40 personas dicen que poco y una minima parte que corresponde a 8 personas respondieron que no atribuyen en nada sus estado de animo a la situación de aislamiento.

Es decir que la gran mayoría correspondiente al $92 \%$ atribuyen sus estados de ánimo al estado de emergencia sanitaria y humanitaria decretado por la pandemia.

\section{Coeficiente correlacional de Pearson}

De los resultados se obtuvo la consideración de que entre la variable independiente que es la resiliencia y la dependiente que son los síntomas depresivos, existe una correlación negativa moderada, es decir que al incrementar una variable la otra se ve disminuida. En esta investigación los valores de resiliencia en su mayoría comprenden entre los valores promedios (44\%) y el alto (42\%) a su vez, la mayor cantidad de muestra obtuvo niveles mínimos de síntomas depresivos (79\%) Entonces se confirma que existe una correlación negativa moderada entre las variables estudiadas en la presente investigación.

En cuanto al alcance esta investigación se llevó a cabo con base en varios objetivos, en primera instancia conocer los niveles de resiliencia de la muestra estudiada, y se diría que gracias a la aplicación de la Escala de Resiliencia de Saavedra y Villalta se dio respuesta al mismo, pues se determinó que existen múltiples escenarios en cuanto a los niveles de resiliencia; se obtuvieron frecuencias para los intervalos de nivel bajo, promedio y alto, cabe enfatizar que estas dos últimas obtuvieron mayores frecuencias, que el primero.

Otro de los objetivos fue evaluar la presencia de síntomas depresivos, mismo que se logró gracias a la aplicación del inventario de depresión de Beck II, y la complementación del mismo a través de la entrevista semiestructurada, cuya finalidad fue conocer aspectos cognitivos y emocionales de los estudiantes con respecto a la situación actual de pandemia. Se conoció que existen diversos síntomas depresivos, sin embargo, el mayor porcentaje de la muestra corresponde a presentar una sintomatología mínima.

Correlacionar estas dos variables fue el tercer objetivo, el cual se dio por cumplido por la aplicación del método del Coeficiente correlacional de Pearson. Allí se observó una correlación negativa moderada, en la que los participantes presentaron valores que se consideran promedio y alto en resiliencia. Diferente a esto, las mayores frecuencias corresponden a niveles mínimos y leves de síntomas depresivos en los estudiantes.

\section{CONCLUSIONES}

En el proceso de investigación se identificaron los niveles de resiliencia y síntomas depresivos en los estudiantes participantes, durante el periodo pandémico, lo mismo que permitió concluir lo siguiente:

Al ser la resiliencia una capacidad que se puede desarrollar y al estar presente en los estudiantes, permite la consecución de las actividades cotidianas a pesar de los cambios evidentes a los que ha sido expuesta la población. La muestra estudiada presenta en su mayoría niveles de resiliencia promedio y alto, lo que evidencia que en alguna medida estos estudiantes han desarrollado esta capacidad, lo que les favoreció la adaptación frente a la realidad circundante. Por el contrario se encuentra la muestra minoritaria que presentan niveles de resiliencia bajos, lo cual puede conllevar a un estancamiento y no superación del estado actual.

Ante el desconocimiento y sobreinformación del COVID-19, muchos estudiantes han visto comprometido de forma negativa sus expectativas futuras, lo cual incrementa sentimientos de desesperanza que dan cabida a esta sintomatología, 
que altera el estado emocional y no permite llevar a cabo proyectos de vida ya establecidos.

Los resultados expuestos estadísticamente a través del instrumento de medición de la depresión (Inventario de depresión de Beck II) revelan la presencia de síntomas depresivos moderados y graves en un porcentaje reducido de la muestra estudiada, lo que no debe pasar desapercibido, más aun tomando en cuenta que se trata de futuros profesionales de salud mental, y que frente a esta situación es posible que realicen prácticas con personas que vivencian en menor o mayor medida estas emociones.

La evidencia obtenida a través de la aplicación de la fórmula del Coeficiente correlacional de Pearson, muestra la correlación negativa que existe entre las variables presentadas, lo que establece directamente que a mayor resiliencia disminuyen los síntomas depresivos; no obstante, con la consecución de la problemática actual, se corre el riesgo de generar la disminución de la resiliencia y por consecuencia el aumento de síntomas depresivos.

\section{REFERENCIAS}

El Universo. (2020). Pandemia de coronavirus afecta de forma significativa la salud mental de los ecuatorianos, según estudio. https://www.eluniverso.com/ larevista/2020/07/06/nota/7897557/ pandemia-coronavirus-impacta-saludmental-ecuatorianos-estudio

Infobae. (2020). COVID-19: un tercio de las personas muestra signos de ansiedad o depresión clínica. Argentina: Infobae. https://www.infobae.com/america/ tendencias-america/2020/05/27/covid-19un-tercio-de-las-personas-muestra-signosde-ansiedad-o-depresion-clinica/
López, A. (2015). Factores predictores de procesos resilientes en jóvenes universitarios. (Tesis doctoral). Universidad de Coruña, Galicia, España. https://n9.cl/ $\mathrm{kd} \times 87$

Organización Mundial de la Salud. (2020). Depresión. https://www.who.int/es/newsroom/fact-sheets/detail/depression

Ozamiz, N., Dosil, M., Picaza, M., y Idoiaga, N. (2020). Niveles de estrés, ansiedad y depresión en la primera fase del brote del COVID-19 en una muestra recogida en el norte de España. Scielo, 36(4). https:// www.scielo.br/scielo.php?script $=$ sci_ arttext\&pid=S0102-311X2020000405013

Programa de las Naciones Unidas para el Desarrollo. (2020). COVID-19: La pandemia. La humanidad necesita liderazgo y solidaridad para vencer a COVID-19. Ecuador: PNUD. https://www.ec.undp.org/ content/ecuador/es/home/coronavirus. html

Saavedra, E., y Villalta, M. (2008). Medición de las características resilientes, un estudio comparativo en personas entre 15 y 65 años. Liberabit, 14(14). https://www.researchgate. net/publication/28235553_Medicion_ de_las_caracteristicas_resilientes_un_ estudio_comparativo_en_personas_ entre_15_y_65_anos

Schofield, M., O'Halloran, P., Mclean, S. y Forrester-Knauss, C. (2016). Depressive Symptoms Among Australian University Students: Who Is at Risk?. Australian Psychologist, 51(2), pp. 135-144. https:// psycnet.apa.org/record/2016-14477-009

Viejo, M. (2020). Uno de cada cinco españoles sufre síntomas de depresión y ansiedad por el coronavirus. Madrid: El País. https:// elpais.com/espana/madrid/2020-05-06/ uno-de-cada-cinco-espanoles-sufresintomas-de-depresion-y-ansiedad-por-elcoronavirus.html 